\title{
A new positivity condition for the curvature of Hermitian manifolds
}

\author{
Freid Tong
}

\begin{abstract}
In this note, we introduce a new type of positivity condition for the curvature of a Hermitian manifold, which generalizes the notion of nonnegative quadratic orthogonal bisectional curvature to the non-Kähler case. We derive a Bochner formula for closed $(1,1)$-forms from which this condition appears naturally and prove that if a Hermitian manifold satisfy our positivity condition, then any class $\alpha \in H_{B C}^{1,1}(X)$ can be represented by a closed $(1,1)$-form which is parallel with respect to the Bismut connection. Lastly, we show that such a curvature positivity condition holds on certain generalized Hopf manifolds and on certain Vaisman manifolds.
\end{abstract}

\section{Introduction}

A general principle in Kähler geometry has been that positive curvature conditions impose important geometric and topological constraints on the manifold. A manifestation of this is the solution of the Frankel conjecture by Siu-Yau and Mori [24, 13], and its generalization by Mok [11], which can be viewed as a uniformization theorem for Kähler manifolds with nonnegative bisectional curvature. In recent years, the study of non-Kähler Hermitian geometry has received a lot of attention, partly due to its connection with heterotic string theory $[4,6,7,21,22]$. It is then natural to look for curvature positivity conditions in nonKähler geometry which can lead to significant geometric and topological consequences. In one such direction, Ustinovskiy [28] proposes to study the uniformization of Hermitian manifolds with nonnegative Griffiths curvature by using a geometric flow which preserves the positivity of the Griffiths curvature. As shown by Fei and Phong [5], the Hermitian curvature flow which he identified as positive curvature preserving is precisely the same as the flows motivated by string theory introduced in [22].

In this article, we introduce a new type of curvature positivity condition involving a tensor $Q$, which is different from the Chern curvature. This tensor $Q$ is less positive than the Chern curvature, hence the corresponding positivity conditions formulated using $Q$ are generally stronger than the corresponding positivity of the Chern curvature. We

This work is supported in part by NSF grant DMS-1855947. 
prove a general Bochner-Kodaira type formula, in which this tensor appears naturally and we show that under a certain $Q$-nonnegativity condition, every class in $\alpha \in H_{B C}^{1,1}(X)$ can be represented by a closed $(1,1)$-form which is parallel with respect to the Bismut connection. This can be seen as a generalization of a theorem by Howard-Smyth-Wu [10] to the non-Kähler setting. Perhaps surprisingly, $Q$ also arises in several different contexts

in non-Kähler geometry: on one hand, it can be interpreted as a combination of the form $\partial \bar{\partial} \omega$ with the $(1,1)$-part of the curvature of the Bismut connection. On the other hand, it acquires an additional symmetry on Vaisman manifolds. We discuss some corresponding applications, which suggest that $Q$ may be very useful for future investigations in nonKähler geometry.

\subsection{Background and conventions}

Let $(X, J, g)$ be a compact Hermitian manifold, then in local holomorphic coordinates, we can write $\omega=\sum_{i, j} g_{i \bar{j}} \sqrt{-1} d z^{i} \wedge d \bar{z}^{j}$.

On the holomorphic tangent bundle, there is a natural connection called the Chern connection, which in holomorphic coordinate is

$$
\begin{aligned}
& \nabla_{i} X^{j}=\partial_{i} X^{j}+\Gamma_{i l}^{j} X^{l} \\
& \nabla_{\bar{i}} X^{j}=\partial_{\bar{i}} X^{j}
\end{aligned}
$$

where the connection coefficients are given by

$$
\Gamma_{k i}^{j}=g^{j \bar{m}} \partial_{k} g_{\bar{m} i}
$$

The Torsion tensor of the Chern connection is $T(X, Y)=\nabla_{X} Y-\nabla_{Y} X-[X, Y]$ and it satisfies $T(J X, Y)=T(X, J Y)$. Hence the torsion has no $(1,1)$-component, and it is entirely determined by its $(2,0)$-component. In local holomorphic coordinates, this is given by

$$
T_{i j}^{k}=\Gamma_{i j}^{k}-\Gamma_{j i}^{k}
$$

which is zero iff the metric is Kähler.

The curvature of the Chern connection satisfies the symmetry

$$
R(X, Y, W, Z)=R(J X, J Y, W, Z)=R(X, Y, J W, J Z)
$$

this implies the curvature has no $(2,0)$ or $(0,2)$ components and the entire curvature tensor is determined by the $(1,1)$-part, which in holomorphic coordinates is given by

$$
R_{i \bar{j} k}^{l}=-\partial_{\bar{j}} \Gamma_{i k}^{l}
$$

We remark that the Chern connection can be characterized as the unique connection such that is Hermitian i.e $\nabla g=0$ and satisfy $\nabla^{0,1}=\bar{\partial}_{T^{1,0} M}$ where $\nabla^{0,1}$ is the $(0,1)$ part of the connection and $\bar{\partial}_{T^{1,0} M}$ is the d-bar operator on holomorphic vector bundles. 


\subsection{Positivity condition}

For this paper, we are interested in a 4 -tensor $Q$, which is defined by the following

$$
Q_{i \bar{j} k \bar{l}}=R_{i \bar{j} k \bar{l}}-g^{p \bar{q}} T_{k p \bar{j}} \overline{T_{l q \bar{i}}}
$$

It turns out that this tensor naturally appears in a Bochner formula for closed $(1,1)$-forms and has a nice connection with the Bismut connection.

Remark 1.1. It is also worth noting that the contraction of $Q$ in the first two coordinates coincides with the evolution term in the pluriclosed flow defined by Streets and Tian in [25], the same term also appears in the Anomaly flow in dimension three which is studied by Phong, Picard, Zhang in [22].

We now state the positivity condition that we are interested in, this new positivity condition is expressed in terms of the $Q$ tensor.

Definition 1.1. We say $(X, J, g)$ is $Q$-nonnegative if in any orthonormal frame (i.e where $\left.g_{i \bar{j}}=\delta_{i j}\right)$ and for any list of real numbers $\lambda_{1}, \ldots, \lambda_{n}$, we have

$$
\sum_{k, m=1}^{n} Q_{m \bar{m} k \bar{k}}\left(\lambda_{k}^{2}-\lambda_{k} \lambda_{m}\right) \geq 0
$$

Remark 1.2. If $Q$ satisfies an additional symmetry $Q_{i \bar{j} k \bar{l}}=Q_{k \bar{l} \bar{j}}$, then this expression simplifies

$$
2 \sum_{k, m=1}^{n} Q_{m \bar{m} k \bar{k}}\left(\lambda_{k}^{2}-\lambda_{k} \lambda_{m}\right)=\sum_{k, m=1}^{n} Q_{m \bar{m} k \bar{k}}\left(\lambda_{k}-\lambda_{m}\right)^{2}
$$

This is the case when the metric is Kähler, in that case $Q=R$ and the condition reduces to the nonnegative quadratic orthogonal bisectional curvature condition. (see [3])

Remark 1.3. A simple observation is that if a product manifold $M \times N$ is $Q$-nonnegative, then each of its components must be $Q$-nonnegative.

Example 1.4. All Bismut-flat manifold are $Q$-nonnegative. Indeed, In it is shown in [32] that these manifold have to be pluriclosed, and from the discussion in section 2, it follows that on these manifolds, $Q$ is equal to the curvature of the Bismut connection which is 0, hence Bismut-flat manifolds are all $Q$-nonnegative. These manifolds has been classified in [31], and their universal covers must a product of a compact semisimple Lie group with a real vector space.

We will give several other examples where the positivity condition holds in Section 3.

We now state our main theorem regarding manifolds with $Q$-nonnegative $Q$ tensors. Our theorem implies that on a $Q$-nonnegative manifold, one can always solve the PoincareLelong equation for a closed $(1,1)$-form up to the addition of a Bismut parallel $(1,1)$-form.

Theorem 1.5. Suppose $(X, J, g)$ is a compact Hermitian manifold which is Q-nonnegative, then any closed $(1,1)$-form with constant trace is parallel with respect to the Bismut connection. In particular, any class in the Bott-Chern cohomology $H_{B C}^{1,1}(X)$ contains a representative which is parallel respect to the Bismut connection. 
Our theorem can be viewed as a non-Kähler generalization of a theorem of HowardSmyth-Wu [10], which says for a compact Kähler manifold with non-negative quadratic orthogonal bisectional curvature, any class in $H^{1,1}(X)$ contains a parallel representative given by the unique harmonic form in that class. This turned out to be a key ingredient of the resolution of the generalized Frankel conjecture by Mok [11]. The key to the proof of the theorem is a Bochner-Kodaira type identity for closed $(1,1)$-form, which generalizes the identity in [10]. In the Kähler case, such an identity was also used by Mok-Siu-Yau [12] to study the solution Poincare-Lelong equation in relation to the uniformization conjecture for non-compact Kähler manifolds. We refer the readers to $[14,15]$ and the references therein for subsequent developments in that direction.

\section{$2 \quad \mathrm{Q}$ and the Bismut connection}

On a Hermitian manifold, there is another canonical connection called the Bismut connection, this is also sometimes called the Strominger connection or the Strominger-Bismut connection in literature. This connection was first written down by physicists (see [27, 9]) and Bismut rediscovered and used it in [2] to prove an index formula for non-Kähler manifolds. This connection has received a lot of attention recently because of its relation to the study of non-Kähler Calabi-Yau manifolds, and the Hull-Strominger system arising from physics, see [6] and [23] where the Bismut connection is used in an crucial way. It is also a natural connection to in the study of pluriclosed metrics via the geometric flow as defined by Streets and Tian [25, 26].

Definition 2.1 (Bismut connection). The Bismut connection is the connection on $T^{1,0} M$ which in local holomorphic coordinates is given by

$$
\begin{aligned}
\nabla_{i}^{+} X^{j} & =\partial_{i} X^{j}+\Gamma_{l i}^{j} X^{l} \\
\nabla_{\bar{i}}^{+} X^{j} & =\partial_{\bar{i}} X^{j}+g^{j \bar{m}} \overline{T_{i m \bar{l}}} X^{l}
\end{aligned}
$$

where $\Gamma_{k i}^{j}=g^{j \bar{m}} \partial_{k} g_{\bar{m} i}$ and $T_{i m \bar{l}}=\partial_{i} g_{m \bar{l}}-\partial_{m} g_{i \bar{l}}$.

Remark 2.1. One can check that this connection respects both the metric and the complex structure (i.e $\nabla^{+} g=\nabla^{+} J=0$ ) and the corresponding Torsion tensor $T^{+}(X, Y, Z)=$ $g\left(\nabla_{X}^{+} Y-\nabla_{Y}^{+} X-[X, Y], Z\right)$ is skew-symmetric. These properties uniquely characterizes the Bismut connection.

The curvature of the Bismut connection does not satisfy the same symmetries as the curvature of the Chern connection. Thus in general, the curvature has a decomposition into $(2,0),(1,1)$ and (0,2)-parts, and we compute the (1,1)-part of the curvature of the Bismut connection in terms of the curvature and torsion of the Chern connection.

Lemma 2.2. The $(1,1)$-part of the curvature of the Bismut connection is given by

$$
B_{i \bar{j} k \bar{l}}=R_{k \bar{j} i \bar{l}}-R_{i \bar{j} k \bar{l}}+R_{i \bar{l} k \bar{j}}-T_{k i \bar{\gamma}} \overline{T_{j l}^{\gamma}}-g^{\gamma \bar{\kappa}} T_{i \gamma \bar{l}} \overline{T_{j \kappa \bar{k}}}
$$


where $B$ is the curvature of the Bismut connection and $R$ is the curvature of the Chern connection.

Proof. The connection coefficients of the Bismut connection is given by

$$
\begin{gathered}
\mathcal{A}_{i k}^{l}=\Gamma_{k i}^{l} \\
\mathcal{A}_{\bar{j} k}^{l}=g^{l \bar{q} \overline{T_{j q \bar{k}}}}
\end{gathered}
$$

and the (1,1)-part of the curvature is given by

$$
\begin{aligned}
B_{i \bar{j} m}^{k} & =\partial_{i} \mathcal{A}_{\bar{j} m}^{k}-\partial_{\bar{j}} \mathcal{A}_{i m}^{k}+\mathcal{A}_{i l}^{k} \mathcal{A}_{\bar{j} m}^{l}-\mathcal{A}_{\overline{j l}}^{k} \mathcal{A}_{i m}^{l} \\
& =g^{k \bar{l}} \nabla_{i} \overline{T_{j l \bar{m}}}+R_{m_{\bar{j} i}{ }^{k}}-T_{i l}^{k} \mathcal{A}_{\bar{j} m}^{l}+\mathcal{A}_{\overline{j l}}^{k} T_{i m}^{l} \\
& =g^{k \bar{l}} R_{i \bar{l} m \bar{j}}-R_{i \bar{j} m}^{k}+R_{m \bar{j} i}^{k}-g^{l \bar{n}} T_{i l}^{k} \overline{T_{j n \bar{m}}}+g^{k \bar{n}} \overline{T_{j n \bar{l}}} T_{i m}^{l}
\end{aligned}
$$

lowering the last index gives the result.

Proposition 2.3. The following formula holds

$$
Q_{i \bar{j} k \bar{l}}=B_{k \bar{l} \bar{j} \bar{j}}+\partial_{\bar{l}} T_{i k \bar{j}}-\partial_{\bar{j}} T_{i k \bar{l}}
$$

where $B_{k \bar{l} i \bar{j}}$ is the $(1,1)$ part of the curvature of the Bismut connection.

proof of Proposition 2.3. In local coordinates where $g_{i \bar{j}}=\delta_{i j}$ and $\partial_{i} g_{j \bar{l}}=\frac{1}{2} T_{i j \bar{l}}$, we compute

$$
\begin{aligned}
\partial_{\bar{l}} T_{k i \bar{j}}-\partial_{\bar{j}} T_{k i \bar{l}} & =\partial_{\bar{l}} \partial_{k} g_{i \bar{j}}-\partial_{\bar{l}} \partial_{i} g_{k \bar{j}}-\partial_{k} \partial_{\bar{j}} g_{i \bar{l}}+\partial_{\bar{j}} \partial_{i} g_{k \bar{l}} \\
& =R_{i \bar{l} k \bar{j}}+R_{k \bar{j} i \bar{l}}-R_{i \bar{j} k \bar{l}}-R_{k \bar{i} \bar{j}}-\sum_{\gamma} T_{k i \bar{\gamma}} \overline{T_{j l \bar{\gamma}}} \\
& =B_{i \bar{j} k \bar{l}}-Q_{k \bar{l} \bar{j}}
\end{aligned}
$$

Remark 2.4. The extra term $\partial_{\bar{l}} T_{i k \bar{j}}-\partial_{\bar{j}} T_{i k \bar{l}}$ is the components of the tensor $\partial \bar{\partial} \omega$, and the previous lemma implies that if $\omega$ is pluriclosed, then $Q$ is simply the $(1,1)$ part of the curvature of the Bismut connection.

\section{$3 \quad \mathrm{Q}$ and a Bochner-Kodaira identity}

In this section, we prove Theorem 1.5. The key is a Bochner-Kodaira type formula for $(1,1)$-forms, from which the tensor $Q$ naturally emerges.

In the Kähler case, this formula is well-known and is intimately related to the uniformization of manifolds with nonnegative bisectional curvature. It first appeared in [1], and is used in [10] to deduce a splitting theorem for manifolds with nonnegative bisectional curvature, which was one of the key imput in the solution of the generalized Frankel conjecture by Mok [11]. 
Theorem 3.1. The following formula holds for $\rho$ a real closed $(1,1)$-form with $g^{i \bar{j}} \rho_{i \bar{j}}=$ const,

$$
g^{i \bar{j}} \partial_{i} \partial_{\bar{j}}|\rho|^{2}=2\left|\nabla^{+} \rho\right|^{2}+2 Q_{i \bar{j} k \bar{l}}\left(g^{i \bar{j}}\left(\rho^{2}\right)^{k \bar{l}}-\rho^{i \bar{j}} \rho^{k \bar{l}}\right)
$$

where $Q_{i \bar{j} k \bar{l}}=R_{i \bar{j} k \bar{l}}-g^{p \bar{q}} T_{k p \bar{j}} \overline{T_{l q} \bar{i}}$.

Proof. In local holomorphic coordinates, we have $\rho=i \rho_{k \bar{j}} d z^{k} \wedge d \bar{z}^{j}$ where $\overline{\rho_{k \bar{j}}}=\rho_{j \bar{k}}$. By the closedness of $\rho$, we have

$$
\nabla_{i} \rho_{k \bar{j}}=\nabla_{k} \rho_{i \bar{j}}+T_{k i}^{m} \rho_{m \bar{j}}
$$

and

$$
\nabla_{\bar{j}} \rho_{i \bar{l}}=\nabla_{\bar{l}} \rho_{i \bar{j}}+\overline{T_{l j}^{n}} \rho_{i \bar{n}}
$$

we compute

$$
\begin{aligned}
\nabla_{i} \nabla_{\bar{j}} \rho_{k \bar{l}}= & \nabla_{i} \nabla_{\bar{l}} \rho_{k \bar{j}}+\nabla_{i}\left[\overline{T_{l j}^{m}} \rho_{k \bar{m}}\right] \\
= & \nabla_{\bar{l}} \nabla_{i} \rho_{k \bar{j}}+\left[\nabla_{i}, \nabla_{\bar{l}}\right] \rho_{k \bar{j}}+\overline{T_{l j}^{m}} \nabla_{i} \rho_{k \bar{m}}+\left[\overline{R_{j \bar{l} l}^{m}}-\overline{R_{l \bar{i} j}^{m}}\right] \rho_{k \bar{m}} \\
= & \nabla_{\bar{l}} \nabla_{k} \rho_{i \bar{j}}+\nabla_{\bar{l}}\left[T_{k i}^{m} \rho_{m \bar{j}}\right]+\left[\nabla_{i}, \nabla_{\bar{l}}\right] \rho_{k \bar{j}}+\overline{T_{l j}^{m}} \nabla_{i} \rho_{k \bar{m}}+\left[\overline{R_{j \bar{i} l}^{m}}-\overline{R_{l \bar{i} j}^{m}}\right] \rho_{k \bar{m}} \\
= & \nabla_{\bar{l}} \nabla_{k} \rho_{i \bar{j}}+T_{k i}^{m} \nabla_{\bar{l}} \rho_{m \bar{j}}+\overline{T_{l j}^{m}} \nabla_{i} \rho_{k \bar{m}}+\left[R_{i \bar{l} \bar{m}}^{m}-R_{k \bar{l} \bar{l}}^{m}\right] \rho_{m \bar{j}}+\left[\overline{R_{j \bar{l} l}^{m}}-\overline{R_{l i \bar{j}}^{m}}\right] \rho_{k \bar{m}} \\
& \quad-R_{i \bar{l} k}^{m} \rho_{m \bar{j}}+R_{i \bar{l} \bar{j}}^{{ }^{\bar{n}}} \rho_{k \bar{n}} \\
= & \nabla_{\bar{l}} \nabla_{k} \rho_{i \bar{j}}+T_{k i}^{m} \nabla_{\bar{l}} \rho_{m \bar{j}}+\overline{T_{l j}^{m}} \nabla_{i} \rho_{k \bar{m}}-R_{k \overline{l i}}^{m} \rho_{m \bar{j}}+R_{i \bar{j} \bar{l}}^{\bar{m}} \rho_{k \bar{m}}
\end{aligned}
$$

differentiating $|\rho|^{2}$ once, we get

$$
\partial_{\bar{j}}|\rho|^{2}=\nabla_{\bar{j}}\left(g^{p \bar{q}} g^{k \bar{l}} \rho_{k \bar{q}} \rho_{p \bar{l}}\right)=g^{p \bar{q}} g^{k \bar{l}}\left(\nabla_{\bar{j}} \rho_{k \bar{q}} \rho_{p \bar{l}}+\nabla_{\bar{j}} \rho_{p \bar{l}} \rho_{k \bar{q}}\right)=2 g^{p \bar{q}} g^{k \bar{l}} \nabla_{\bar{j}} \rho_{k \bar{q}} \rho_{p \bar{l}}
$$

taking the divergence and using our previous calculation for $\nabla_{i} \nabla_{\bar{j}} \rho_{k \bar{q}}$ gives

$$
\begin{aligned}
\Delta|\rho|^{2}= & 2|\nabla \rho|^{2}+2 g^{p \bar{q}} g^{k \bar{l}} g^{i \bar{j}} \nabla_{i} \nabla_{\bar{j}} \rho_{k \bar{q}} \rho_{p \bar{l}} \\
= & 2|\nabla \rho|^{2}+2\langle\partial \bar{\partial} \operatorname{tr} \rho, \rho\rangle+2 g^{p \bar{q}} g^{k \bar{l}}\left[g^{i \bar{j}}\left(T_{k i}^{m} \nabla_{\bar{q}} \rho_{m \bar{j}}+\overline{T_{q j}^{m}} \nabla_{i} \rho_{k \bar{m}}\right)\right] \rho_{p \bar{l}} \\
& \quad+2 g^{k \bar{l}_{12}} \operatorname{tr}_{12}(R)^{\bar{m} p} \rho_{k \bar{m}} \rho_{p \bar{l}}-2 R^{\bar{l} p \bar{j} m} \rho_{m \bar{j}} \rho_{p \bar{l}}
\end{aligned}
$$

We now specialize to a coordinate system where $g_{i \bar{j}}=\delta_{i \bar{j}}$ and $\rho_{i \bar{j}}=\lambda_{i} \delta_{i \bar{j}}$, and use the assumption $g^{i \bar{j}} \rho_{i \bar{j}}=$ const,

$$
\Delta|\rho|^{2}=2\left|\nabla_{i} \rho\right|^{2}+\sum(\cdots)+2 \sum_{k, m=1}^{n} R_{m \bar{m} k \bar{k}}\left(\lambda_{k}^{2}-\lambda_{k} \lambda_{m}\right)
$$

where

$$
\begin{aligned}
\sum(\cdots) & =2 \sum_{k, m, i=1}^{n}\left[T_{k i \bar{m}} \nabla_{\bar{k}} \rho_{m \bar{i}}+\overline{T_{k i \bar{m}}} \nabla_{i} \rho_{k \bar{m}}\right] \lambda_{k} \\
& =2 \sum_{i, k, m=1}^{n}\left[T_{k i \bar{m}} \nabla_{\bar{k}} \rho_{m \bar{i}}+\overline{T_{k i \bar{m}}} \nabla_{k} \rho_{i \bar{m}}\right] \lambda_{k}+2 \sum_{i, k, m=1}^{n} \overline{T_{k i \bar{m}}} T_{k i \bar{m}} \lambda_{m} \lambda_{k}
\end{aligned}
$$


we can complete the square for the gradient term and we get

$$
\begin{aligned}
\Delta|\rho|^{2}= & 2 \sum_{i, k, m=1}^{n}\left|\nabla_{k} \rho_{i \bar{m}}+T_{k i \bar{m}} \lambda_{k}\right|^{2}+2 \sum_{i, k, m=1}^{n}\left|T_{k i \bar{m}}\right|^{2} \lambda_{m} \lambda_{k} \\
& -2 \sum_{k, i, m=1}^{n}\left|T_{k i \bar{m}}\right|^{2} \lambda_{k}^{2}+2 \sum_{k, m=1}^{n}\left(R_{m \bar{m} k \bar{k}}\left(\lambda_{k}^{2}-\lambda_{k} \lambda_{m}\right)\right) \\
= & 2 \sum_{i, k, m=1}^{n}\left|\nabla_{k} \rho_{i \bar{m}}+T_{k i \bar{m}} \lambda_{k}\right|^{2}-2 \sum_{k, m=1}^{n}\left(R_{m \bar{m} k \bar{k}}-\sum_{i=1}^{n} T_{k i \bar{m}} \overline{T_{k i \bar{m}}}\right) \lambda_{k} \lambda_{m} \\
& +2 \sum_{k, m=1}^{n}\left(R_{m \bar{m} k \bar{k}}-\sum_{i=1}^{n} T_{k i \bar{m}} \overline{T_{k i \bar{m}}}\right) \lambda_{k}^{2} \\
= & 2 \sum_{i, k, m=1}^{n}\left|\partial_{i} \rho_{k \bar{m}}-\Gamma_{k i \bar{m}} \lambda_{m}-T_{i k \bar{m}} \lambda_{k}\right|^{2}+2 \sum_{k, m=1}^{n}\left(R_{m \bar{m} k \bar{k}}-\sum_{i=1}^{n} T_{k i \bar{m}} \overline{T_{k i \bar{m}}}\right) \\
= & \left.2\left|\nabla^{+} \rho\right|^{2}+2 \sum_{k, m=1}^{2} \lambda_{k} \lambda_{m}\right) \\
&
\end{aligned}
$$

where $Q_{i \bar{j} k \bar{l}}=R_{i \bar{j} k \bar{l}}-g^{p \bar{q}} T_{k p \bar{j}} \overline{T_{l q \bar{i}}}$.

Corollary 3.2. Suppose $(X, J, g)$ is $Q$-nonnegative, then any closed real $(1,1)$-form $\rho$ with constant trace is parallel with respect to the Bismut connection. Moreover, $T(X, Y, \bar{Z})=0$ if $X, Y, \bar{Z}$ are in different eigenspaces of $\rho$ corresponding to eigenvalues $\lambda_{X}, \lambda_{Y}, \lambda_{Z}$ and $\lambda_{X}+\lambda_{Y}-\lambda_{Z} \neq 0$.

Proof. If $(M, J, g)$ is $Q$-nonegative, then we have

$$
\Delta|\rho|^{2} \geq 2\left|\nabla^{+} \rho\right|^{2} \geq 0
$$

and by the maximum principle, we must have $|\rho|^{2}=$ const which implies $\Delta|\rho|^{2}=0$ so the right hand side of equation 17 must be identically 0 , hence we have $\nabla^{+} \rho=0$. If we work in coordinates where $g_{i \bar{j}}=\delta_{i j}$ and $\rho_{i \bar{j}}=\lambda_{i} \delta_{i j}$, then $\nabla^{+} \rho=0$ and $\rho$ being closed implies that

$$
T_{k i \bar{m}}\left(\lambda_{k}+\lambda_{i}-\lambda_{m}\right)=0
$$

from which the second part of the proposition follows.

Proof of Theorem 1.5. The first statement follows by Corollary 3.2, we will prove the second statement that every class in $H_{B C}^{1,1}(X)$ contains a Bismut parallel representative. Recall that

$$
H_{B C}^{1,1}(X):=\frac{\left\{\alpha \in \Omega^{1,1} \mid d \alpha=0\right\}}{i \partial \bar{\partial} C^{\infty}(X, \mathbb{C})},
$$


hence this statement amounts to showing that for any closed $(1,1)$-form $\alpha$, there exist a smooth function $u$ such that $\nabla^{+}(\alpha+i \partial \bar{\partial} u)=0$. Let $\alpha$ be a real closed $(1,1)$-form. In [8], Gauduchon proved that every Hermitian metric is conformally equivalent to a Gauduchon metric, so let $\hat{\omega}=e^{f} \omega$ be a Gauduchon metric in the conformal class of $\omega$. By the Gauduchon property, we know the equation $i \partial \bar{\partial} u \wedge \hat{\omega}^{n-1}=f \hat{\omega}^{n}$ has a solution iff $\int_{X} f \hat{\omega}^{n}=0$. Let $u$ solve the equation

$$
n \frac{\sqrt{-1} \partial \bar{\partial} u \wedge \hat{\omega}^{n-1}}{\hat{\omega}^{n}}=\operatorname{tr}_{\hat{\omega}} \alpha+c e^{-f}
$$

where $c$ is the constant given by

$$
c=-\frac{\int_{X}\left(\operatorname{tr}_{\hat{\omega}} \alpha\right) \hat{\omega}^{n}}{\int_{X} e^{-f} \hat{\omega}^{n}}
$$

Setting $\rho=\alpha-\sqrt{-1} \partial \bar{\partial} u$, then $d \rho=0$ and $\operatorname{tr}_{\omega} \rho=\operatorname{tr}_{\omega} \alpha-\Delta_{\omega} u=c$, hence by Corollary 3.2 , we have $\nabla^{+} \rho=0$. If $\alpha$ is not real then we can write $\alpha=u+i v$ where $u, v$ are the real and imaginary parts of $\alpha$, then $u, v$ are both real closed $(1,1)$ forms and we can apply the above argument to both $u$ and $v$.

Corollary 3.3. If $(X, J, g)$ is non-Kähler, $Q$-nonnegative and $H_{B C}^{1,1} \neq 0$. Then the holonomy of $\nabla^{+}$is contained in a subspace $U(m) \times U(n-m) \subset U(n)$.

Proof. Since $H_{B C}^{1,1} \neq 0$, by Theorem 1.5, there exist a class $0 \neq[\rho] \in H_{B C}^{1,1}$ containing a Bismut flat representative $\rho$, and furthermore, since $X$ is non-Kähler, $\rho$ is not a multiple of $g$. Hence if $\lambda_{1}, \ldots, \lambda_{n}$ are the eigenvalues of $\rho$ with respect to $g$, then there exist at least two distinct eigenvalues, and since both $g$ and $\rho$ is flat with respect to the Bismut connection, it follows that the eigenspaces of $\rho$ are invariant under the holonomy $\nabla^{+}$, and we have our result.

\section{Q and Vaisman manifolds}

In this section, we study this positivity condition on a class of Locally conformally Kähler manifolds called Vaisman manifolds. These manifolds were first introduced by Vaisman [29] as an important class of complex non-Kähler manifold which he called generalized Hopf manifolds. By [17, 19], a large class of these manifolds can be viewed as a Sasakian manifolds equipped with a Sasakian automorphism. For more on these manifolds, we refer the reader to $[17,19]$ and the references therein.

Definition 4.1. (1) A compact Hermitian manifold $(M, J, g)$ is called locally conformally Kähler if there exist an closed form $\theta$ such that

$$
d \omega=\theta \wedge \omega
$$

The 1 -form $\theta$ is called the Lee form. 
(2) A locally conformally Kähler manifold is a Vaisman manifold if $\nabla^{L C} \theta=0$, where $\nabla^{L C}$ is the Levi-Civita connection of $(M, J, g)$.

Remark 4.1. A locally conformally Kähler metric can always locally be written as a Kähler metric times a conformal factor, and the coverse is clearly true as well. Indeed, since the Lee form $\theta$ is closed, by the Poincare lemma, it is locally exact, hence locally, we can always write $\theta=-d f$ for some locally defined functin $f$, then $e^{f} \omega$ is a Kähler metric since $d\left(e^{f} \omega\right)=e^{f} d f \wedge \omega+e^{f} d \omega=e^{f}(\theta+d f) \wedge \omega=0$. Globally, any LCK manifold admits a Kähler covering. Indeed, on any cover $\pi: \tilde{M} \rightarrow M$ such that $H^{1}(\tilde{M}, \mathbb{R})=0$, the Lee form on the cover $\pi^{\star} \theta$ is globally exact, and the same argument says that $\pi^{\star} \omega$ is globally conformal to a Kähler metric.

From this discussion, we see that a locally conformally Kähler metric can locally be described by two functions, a Kähler potential for the Kähler metric $\varphi$ and a conformal factor $e^{f}$. On a Vaisman manifolds, if $\theta$ is exact and $\theta=-d f$, then in [30] Verbitsky showed that the metric $\omega$ can be expressed by $e^{-f} i \partial \bar{\partial} e^{f}$. Hence up to a cover, a Vaisman metric can be described by the data of a single potential function $\varphi$ and the metric is given by $\omega=\varphi^{-1} i \partial \bar{\partial} \varphi$. Motivated by this, Ornea and Verbitsky introduced a more general class of metrics called LCK metrics with potential in [18].

Definition 4.2. A locally conformally Kähler manifold $(M, J, g)$ has a potential if it has a Kähler covering $\pi: \tilde{M} \rightarrow M$ with a global positive Kähler potential $\varphi \in C_{>0}^{\infty}(\tilde{M})$ such that $\varphi^{-1} i \partial \bar{\partial} \varphi=c \pi^{\star} \omega$ for some constant $c$.

It turns out that on this class of manifolds, the $Q$-tensor enjoys an extra symmetry that the Chern curvature does not have.

Proposition 4.2. Suppose a Hermitian manifold $(M, J, g)$ is an LCK manifold with potential, then $Q$ has the additional symmetry $Q_{i \bar{j} k \bar{l}}=Q_{k \bar{l} i \bar{j}}$.

To prove this, first we need to compute the change of the $Q$ tensor under a conformal change

Lemma 4.3. If $g$ is a Kähler metric and $\tilde{g}=e^{f} g$, then we have

$$
\tilde{Q}_{i \bar{j} k \bar{l}}=e^{f}\left[R_{i \bar{j} k \bar{l}}-g_{k \bar{l}} f_{i \bar{j}}-f_{k} f_{\bar{l}} g_{i \bar{j}}-|\partial f|^{2} g_{k \bar{j}} g_{i \bar{l}}+f_{k} f_{\bar{j}} g_{i \bar{l}}+f_{i} f_{\bar{l}} g_{k \bar{j}}\right]
$$

Proof. By straightforward computation, we have

$$
\tilde{\Gamma}_{i j}^{k}=\tilde{g}^{k \bar{l}} \partial_{i} \tilde{g}_{j \bar{l}}=e^{f} g^{k \bar{l}} \partial_{i}\left(e^{f} g_{j \bar{l}}\right)=\Gamma_{i j}^{k}+f_{i} \delta_{j}^{k}
$$

since we assumed $g$ is Kähler, we have $\Gamma_{i j}^{k}=\Gamma_{j i}^{k}$, so

$$
\tilde{T}_{i j}^{k}=f_{i} \delta_{j}^{k}-f_{j} \delta_{i}^{k} \Longrightarrow \tilde{T}_{i j \bar{k}}=e^{f}\left(f_{i} g_{j \bar{k}}-f_{j} g_{i \bar{k}}\right)
$$

and

$$
\tilde{R}_{i \bar{j} k}^{m}=-\partial_{\bar{j}} \tilde{\Gamma}_{i k}^{m}=-\partial_{\bar{j}}\left(\Gamma_{i k}^{m}+f_{i} \delta_{k}^{m}\right)=R_{i \bar{j} k}^{m}-f_{i \bar{j}} \delta_{k}^{m}
$$

loweing indices gives

$$
\tilde{R}_{i \bar{j} k \bar{l}}=e^{f}\left(R_{i \bar{j} k \bar{l}}-f_{i \bar{j}} g_{k \bar{l}}\right)
$$

substituting into the formula $\tilde{Q}_{i \bar{j} k \bar{l}}=\tilde{R}_{i \bar{j} k \bar{l}}-\tilde{g}^{p \bar{q}} \tilde{T}_{k p \bar{j}} \tilde{\bar{T}}_{l q \bar{i}}$ the result. 
proof of Proposition 4.2. Suppose $g$ satisfies $g=c \varphi^{-1} d d^{c} \varphi$, then by Lemma 4.3 with $g=$ $i \partial \bar{\partial} \varphi$ and $f=-\log \varphi+\log c$, we get that $Q$ is given by

$Q_{i \bar{j} k \bar{l}}=c \varphi^{-1}\left[R_{i \bar{j} k \bar{l}}^{i \partial \bar{\partial} \varphi}+\frac{\varphi_{k \bar{l}} \varphi_{i \bar{j}}}{\varphi}-\frac{\varphi_{k \bar{l}} \varphi_{i} \varphi_{\bar{j}}}{\varphi^{2}}-\frac{\varphi_{i \bar{j}} \varphi_{k} \varphi_{\bar{l}}}{\varphi^{2}}-|\partial \log \varphi|_{i \partial \bar{\partial} \varphi}^{2} \varphi_{k \bar{j}} \varphi_{i \bar{l}}+\frac{\varphi_{i \bar{l}} \varphi_{k} \varphi_{\bar{j}}}{\varphi^{2}}+\frac{\varphi_{k \bar{j}} \varphi_{i} \varphi_{\bar{l}}}{\varphi^{2}}\right]$

from which we can read off the symmetry $Q_{i \bar{j} k \bar{l}}=Q_{k \bar{l} i \bar{j}}$.

All Vaiman manifolds are LCK with potential, and the Vaisman manifolds are charaterized by the following condition.

Theorem 4.4 ([20]). A compact LCK manifold with potential $(M, J, g)$ is Vaisman if and only if $|\theta|=$ const.

Remark 4.5. Let $(M, J, g)$ be LCK with potential, then if we pull-back the metric $g$ to its Kähler cover and write $\pi^{\star} \omega=c \frac{i \partial \bar{\partial} \varphi}{\varphi}$ for some potential $\varphi$, then the condition $|\theta|=$ const is equivalent to $|\partial \log \varphi|_{\varphi^{-1} i \partial \bar{\partial} \varphi}^{2}=1$.

Theorem 4.6. A Vaisman manifold $(M, J, g)$ is Q-nonnegative if the corresponding Kähler metric $\tilde{\omega}$ on its Kähler cyclic cover has nonnegative quadratic orthogonal bisectional curvature.

Proof. Suppose $M$ is a Vaisman manifold and $\tilde{M} \rightarrow M$ be a Kähler cyclic cover with nonnegative quadratic orthogonal bisectional curvature. Since Vaiman manifolds are LCK with potential, by Proposition 4.2, we know that on a Vaisman manifiold $Q$ satisfy the symmetry $Q_{i \bar{j} k \bar{l}}=Q_{k \bar{l} \bar{j}}$. Moreover, in normal coordinates for $i \partial \bar{\partial} \varphi$, i.e where $\varphi_{i \bar{j}}=\delta_{i \bar{j}}$, and for $m \neq k$, equation 43 reduces to

$$
\begin{aligned}
Q_{m \bar{m} k \bar{k}} & =c \varphi^{-1}\left[R_{m \bar{m} k \bar{k}}^{i \partial \bar{\partial} \varphi}+\frac{1}{\varphi}-\frac{\varphi_{k} \varphi_{\bar{k}}+\varphi_{m} \varphi_{\bar{m}}}{\varphi^{2}}\right] \\
& \geq c \varphi^{-1}\left[R_{m \bar{m} k \bar{k}}^{i \partial \bar{\partial} \varphi}+\frac{1-|\partial \log \varphi|_{\varphi^{-1} i \partial \bar{\partial} \varphi}^{2}}{\varphi}\right]
\end{aligned}
$$

and by the Vaisman condition, we have $|\partial \log \varphi|_{\varphi^{-1} i \partial \bar{\partial} \varphi}^{2}=1$. So in the those coordinates, for any $\lambda_{1}, \ldots, \lambda_{n}$, we get

$$
\sum_{m, k} Q_{m \bar{m} k \bar{k}}\left(\lambda_{m}-\lambda_{k}\right)^{2} \geq c \varphi^{-1} \sum_{m, k} R_{m \bar{m} k \bar{k}}^{i \partial \bar{\partial}}\left(\lambda_{m}-\lambda_{k}\right)^{2} \geq 0
$$

Example 4.7. A diagonal Hopf surface is one which can be written as $M_{\alpha, \beta}=\mathbb{C}^{2} \backslash(0,0) / \sim$ where $\left(z_{1}, z_{2}\right) \sim\left(\alpha z_{1}, \beta z_{2}\right)$ for $|\alpha|=|\beta|<1$. These manifold are diffeomorphic to $S^{3} \times S^{1}$ and are non-Kähler as $b_{1}=1$. They also admit a Vaisman metric given explicitly by

$$
\omega_{M_{\alpha, \beta}}=\frac{4 \sqrt{-1} \partial \bar{\partial}|z|^{2}}{|z|^{2}}
$$


this metric is $Q$-nonegative by Proposition 4.6. In fact this metric is pluriclosed and $Q$ is identically $Q$. One can check that $h_{B C}^{1,1}=1$. Thus Theorem 1.5 implies there exist a Bismut parallel $(1,1)$-form $\rho$, the two eigenspaces of this form are then Bismut parallel subspaces of $T^{1,0}$, hence this gives a splitting of the holomorphic tangent bundle with respect to the Bismut connection. We remark that that Gauduchon and Ornea constructed Vaisman metrics on all class 1 Hopf surfaces in [16], however the Vaiman metrics on the non-diagonal Hopf surfaces are not $Q$-nonnegative. It would be interesting to know if the non-diagonal Hopf surfaces admit other metrics that are $Q$-nonnegative.

Example 4.8. A above construction of a diagonal Hopf surface can be generalized to higher dimensions. Define $M_{\alpha}=\mathbb{C}^{n} \backslash(0, \ldots, 0) / \sim$ where $\alpha=\left(\alpha_{1}, \ldots, \alpha_{n}\right)$ satisfy $\left|\alpha_{1}\right|=\cdots=$ $\left|\alpha_{n}\right|<1$ and $\left(z_{1}, \ldots, z_{n}\right) \sim\left(\alpha_{1} z_{1}, \ldots, \alpha_{n} z_{n}\right)$, then

$$
\omega_{M_{\alpha}}=\frac{4 \sqrt{-1} \partial \bar{\partial}|z|^{2}}{|z|^{2}}
$$

is still $Q$-nonnegative by Proposition 4.6. In the higher dimensional case, the metrics are no longer pluriclosed and $Q$ does not vanish identically.

Acknowledgements: I would like to thank my advisor Duong Phong for many helpful suggestions and for his constant support and encouragement. I am also grateful to Nikita Klemyatin for bringing to my attention the paper [19].

\section{References}

[1] R.L. Bishop and S.I. Goldberg, On the second cohomology group of a Kähler manifold of positive curvature, Proc. Amer. Math. Soc. 16 (1965), 119-122.

[2] J-M. Bismut, A local index theorem for non-Kähler manifolds, Math. Ann. 284 (1989), no. 4, 681-699.

[3] A. Chau and L.F. Tam, On quadratic orthogonal bisectional curvature, J. Differential Geom. 92 (2012), no. 2, 187-200.

[4] T. Fei, Z. Huang and S. Picard, A Construction of Infinitely Many Solutions to the Strominger System, arXiv:1703.10067.

[5] T. Fei and D.H. Phong, Unification of the Kähler-Ricci flow and Anomaly flows, Surveys in Differential Geometry, Vol. 23 (2018), pp. 89-104

[6] T. Fei and S.T. Yau, Invariant solutions to the Strominger system on complex Lie groups and their quotients, Comm. Math. Phys. 338 (2015), no. 3, 1183-1195.

[7] J. Fu and S.T. Yau, The theory of superstring with flux on non-Kähler manifolds and the complex Monge-Ampere equation, J. Differential Geom. 78 (2008), no. 3, 369-428. 
[8] P. Gauduchon, Le théorème de l'excentricité nulle. (French), C. R. Acad. Sci. Paris Sér. A-B 285 (1977), no. 5, A387-A390.

[9] C. Hull and E. Witten, Supersymmetric sigma models and the heterotic string, Physics Letters B, Vol 160, Number 6 (1985), 398-402.

[10] A. Howard, B. Smyth and H. Wu, On compact Kähler manifolds of nonnegative bisectional curvature. I, Acta Math. 147 (1981), no. 1-2, 51-56.

[11] N. Mok. The uniformization theorem for compact Kähler manifolds of nonnegative holomorphic bisectional curvature, J. Differential Geom. 27 (1988), no. 2, 179-214.

[12] N. Mok, Y.T. Siu and S.T. Yau, The Poincaré-Lelong equation on complete Kähler manifolds, Compositio Math. 44 (1981), no. 1-3, 183-218.

[13] S. Mori, Projective manifolds with ample tangent bundles, Ann. of Math. (2) 110 (1979), no. 3, 593-606.

[14] L. Ni and L.F. Tam, Plurisubharmonic functions and the structure of complete Kähler manifolds with nonnegative curvature, J. Differential Geom. 64 (2003), no. 3, 457-524.

[15] L. Ni and L.F. Tam, The Poincaré-Lelong equation via the Hodge-Laplace heat equation, Compos. Math. 149 (2013), no. 11, 1856-1870.

[16] L. Ornea and P. Gauduchon, Locally conformally Kähler metrics on Hopf surfaces, Ann. Inst. Fourier (Grenoble) 48 (1998), no. 4, 1107-1127.

[17] L. Ornea and M. Verbitsky, Structure theorem for compact Vaisman manifolds, Math. Res. Lett. 10 (2003), no. 5-6, 799-805.

[18] L. Ornea and M. Verbitsky, Locally conformal Kähler manifolds with potential, Math. Ann. 348 (2010), no. 1, 25-33.

[19] L. Ornea and M. Verbitsky, LCK rank of locally conformally Kähler manifolds with potential, J. Geom. Phys. 107 (2016), 92-98.

[20] L. Ornea and M. Verbitsky, Hopf surfaces in locally conformally Kähler manifolds with potential, Geometriae Dedicata (2019).

[21] D.H. Phong, Geometric Partial Differential Equations from Unified String Theories, Proceedings of the ICCM 2018, Taipei, Taiwan.

[22] D.H. Phong, S. Picard and X. Zhang, Anomaly flows, Comm. Anal. Geom. 26 (2018), no. 4, 955-1008. 
[23] D.H. Phong, S. Picard and X. Zhang, The Anomaly flow on unimodular Lie groups, Advances in complex geometry, 217-237, Contemp. Math., 735, Amer. Math. Soc., Providence, RI, 2019.

[24] Y.T. Siu and S.T. Yau, Compact Kähler manifolds of positive bisectional curvature, Invent. Math. 59 (1980), no. 2, 189-204.

[25] J. Streets and G. Tian, A parabolic flow of pluriclosed metrics Int. Math. Res. Not. IMRN 2010, no. 16, 3101-3133.

[26] J. Streets and G. Tian, Regularity results for pluriclosed flow Geom. Topol. 17 (2013), no. 4, 2389-2429.

[27] A. Strominger, Superstrings with torsion, Nuclear Phys. B 274 (1986), no. 2, 253-284.

[28] Y. Ustinovskiy, On the Structure of Hermitian Manifolds with Semipositive Griffiths Curvature, Trans. Amer. Math. Soc. (2020)

[29] I. Vaisman, Generalized Hopf manifolds, Geom. Dedicata 13 (1982), no. 3, 231-255.

[30] M. Verbitsky, Theorems on the vanishing of cohomology for locally conformally hyper-Kähler manifolds. (Russian), Tr. Mat. Inst. Steklova 246 (2004), Algebr. Geom. Metody, Svyazi i Prilozh., 64-91; translation in Proc. Steklov Inst. Math. 2004, no. 3(246), 54-78

[31] Q. Wang, B. Yang and F. Zheng, On Bismut flat manifolds, arXiv:1603.07058

[32] Q. Zhao and F. Zheng, Strominger connection and pluriclosed metrics, arXiv:1904.06604

Department of Mathematics, Columbia University, 2990 Broadway, New York, Ny 10027

E-mail address: tong@math.columbia.edu 